\title{
THE GALACTIC KINEMATICS OF MIRA VARIABLES
}

\author{
Michael Feast \\ Astronomy Department, University of Cape Town, Rondebosch,7701, \\ South Africa \\ mwf@artemisia.ast.uct.ac.za
}

\begin{abstract}
The galactic kinematics of Mira variables derived from radial velocities, Hipparcos proper motions and an infrared period-luminosity relation are reviewed. Local Miras in the 145-200day period range show a large asymmetric drift and a high net outward motion in the Galaxy. Interpretations of this phenomenon are considered and (following Feast and Whitelock 2000) it is suggested that they are outlying members of the bulge-bar population and indicate that this bar extends beyond the solar circle.
\end{abstract}

Keywords: stars: AGB: - Galaxy: kinematics and dynamics - Galaxy: structure.

\section{Introduction}

The galactic kinematics of Mira variables have for a long while been of importance in helping us understand both the nature and evolution of Miras as well as the structure of our own Galaxy. This paper is primarily concerned with the second point - what do we learn from Miras about galactic structure? The paper also concentrates on local kinematics, leaving out a detailed discussion of the kinematics of the galactic bulge, much work on which has of course been done here in Japan. It has been possible to take a fresh look at the local kinematics of Miras using Hipparcos astrometry, extensive new infrared photometry and published radial velocities. This was done in a series of three papers (Whitelock, Marang and Feast (2000), paper I: Whitelock and Feast (2000), paper II: Feast and Whitelock (2000a), paper III). The present paper summarizes some of the relevant results from these papers and extends the discussion of the kinematics. 

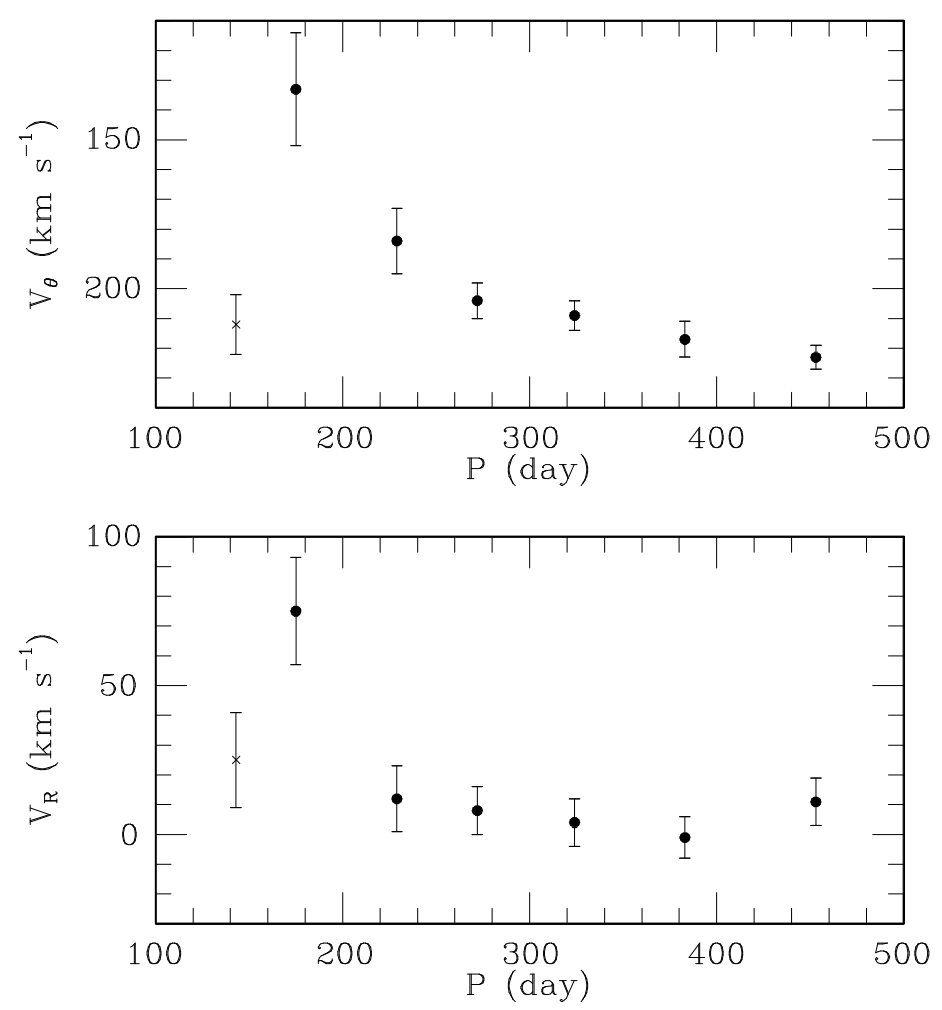

Figure 1. (a) The mean $V_{\theta}$ in various period groups plotted against the mean period of the group. (b) The mean $V_{R}$ in various period groups plotted against the mean period of the group. The cross represents the SP-red variables in both figures.

\section{The Period-Kinematic Sequence}

It has been known for many years that there is a relation between the kinematics and the periods of Mira variables in the general solar neighbourhood. In particular the asymmetric drift and the velocity dispersion increase as one goes from longer to shorter periods. There was however an anomaly. The shortest period group (periods less than $\sim 150$ days) differed strongly from the general trend (Feast 1963). Following an initial study by Hron (1991), it was shown in paper I (see also Whitelock 2002) that combining infrared and Hipparcos magnitudes one sees two sequences of Miras at shorter periods in the period - colour plane, the SP-red and SP-blue stars, and this must be taken into account when discussing the kinematics.

Figure 1a shows a plot of mean velocity of Miras in the direction of galactic rotation, $V_{\theta}$, in period groups. This plot (data in paper III) 
is a distinct improvement on earlier work. It depends on space motions rather than just radial velocities and the SP-red stars have been grouped separately and are denoted by a cross. The figure shows that at the longest periods the stars of the main Mira sequence are moving at close to the circular velocity of galactic rotation, which is taken as $231 \mathrm{~km} . \mathrm{s}^{-1}$ (Feast and Whitelock 1997). There is a clear dependence of mean rotational velocity on period for this main Mira sequence with the short period (145-200 day) Miras having a large asymmetric drift $\left(-97 \pm 20 \mathrm{~km} \cdot \mathrm{s}^{-1}\right)$.

The main Mira sequence now seems reasonably well understood. The infrared colour-period relation for this sequence is the same as that for Miras in globular clusters (paper I and Whitelock 2002), strongly suggesting a similarity between the field and cluster Miras over the period range in common. Not only do the clusters show that their Miras lie at the tip of the AGB, they also show that there is a period-metallicity relation (e.g. Feast and Whitelock 2000b). This allows us to study the galactic kinematics of old populations as a function of metallicity (and possibly also age) over a range of ages and metallicities where there are few if any useful, precise, tracers. For instance globular clusters containing Miras are often classed as "disc" clusters and treated together in kinematic discussions. However the field Miras show that there is a considerable variation of kinematic properties in the period/metallicity range of relevance to these clusters.

Where do the SP-red stars fit in? A full answer to this question may come when more members of the class are identified and when good individual parallaxes are available for a significant number. The best guess at present (see e.g. Whitelock 2002) is that, unlike the stars of the main Mira sequence, they are not at the end of their AGB lives but that they will evolve into longer period Miras of the main Mira sequence.

\section{Local Miras and a Galactic Bar}

A particularly interesting result in paper III is the evidence of a net radial, outward motion $\left(V_{R}\right)$ for Miras in the solar neighbourhood. This is shown as a function of period in figure $1 \mathrm{~b}$ There is evidence of a small outward motion over most of the period range of the Miras. However it is very marked in the group with periods in the range 145 to 200 days where one finds, $V_{R}=+75 \pm 18 \mathrm{~km} . \mathrm{s}^{-1}$ for a mean period of 173 days. Note that in view of the discussion in section 2, the SP-red Miras are omitted in the discussion of the present section.

There has been evidence in the literature for many years that some 
groups of old stars in the solar neighbourhood show a predominant, but modest, outward motion in the Galaxy, though this has not always been recognized. The Hipparcos results, giving parallaxes and proper motions for many thousands of common stars have enabled this to be studied in greater detail. It is now clear that there is a group of old stars with a small asymmetric drift and a modest outward velocity $\left(V_{\theta}=\sim 190 \mathrm{~km} . \mathrm{s}^{-1}, V_{R}=\sim+30 \mathrm{~km} . \mathrm{s}^{-1}\right.$ (Dehnen 1998, 1999, 2000, Fux 2001). Fux has referred to these stars as forming the "Hercules Stream". Dehnen, Fux and also Quillen (2002) each suggest that the stars of the Hercules Stream were originally in circular orbits which have been perturbed into their present state by the influence of a (pre-existing) galactic bar. However, the detailed dynamic processes envisaged differ from author to author.

Fux has suggested that the short period Mira group just discussed belongs to the Hercules Stream. However whilst the Hercules Stream consists of old stars with a wide range of metallicities (Raboud et al. 1998) the large local outward motion in the case of Miras is confined to a restricted range in period, and therefore also in metallicity. The effect is also more extreme than in the Hercules group. This is shown in Figure 2 which plots $V_{\theta}$ against $V_{R}$ for individual Miras in the 145 to 200 day group.

The dotted oval shows the extent of the Hercules Stream as delimited by Dehnen (2000, fig 9). Evidently these Miras do not concentrate in the region of the Hercules Stream.

It is clear that some of the stars in figure 2 are on highly eccentric orbits. About half the sample have perigalactic distances less than about $2 \mathrm{kpc}$ and some at least will pass through the galactic bulge. Despite the small number of stars in this diagram, there is also evidence of a relation between $V_{\theta}$ and $V_{R}$. In paper III we derived the curve shown in the fig 3 which makes the first order assumption that the stars are moving in simple elliptical orbits of different eccentricities but with their major axes aligned. There is then only one free parameter; the position of this major axis with respect to the sun-centre line. The curve is drawn for the best fitting value of this position angle $\left(17_{-4}^{+11}\right.$ degrees). It provides a rather good fit to the data and since this angle is, within the uncertainties, similar to that proposed for the bar in the galactic bulge, we suggested that these 145-200 day Miras in the general solar neighbourhood were outlying members of the bar population itself. If this is the case the bar population extends out to beyond the solar circle.

This discussion depends on the Miras in the general solar neighbourhood and is thus limited in number. In a recent paper Kharchenko et al. (2002) have suggested that over a larger volume of space, the mean $V_{R}$ 


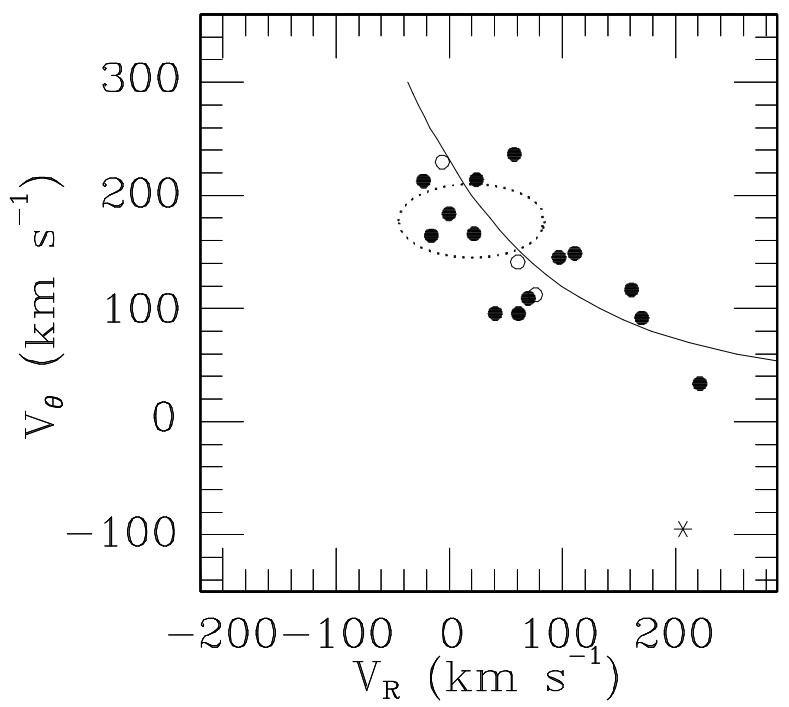

Figure 2. A plot of $V_{\theta}$ against $V_{R}$ for local Miras with periods in the range 145 to 200 days. The open circles denote stars for which the standard error of a velocity component is greater than $20 \mathrm{~km} . \mathrm{s}^{-1}$. The asterisk show the position of S Car which is on a highly eccentric retrograde orbit. The curve and dotted oval are discussed in the text.

for 145-200 day Miras is near zero. More details of their work are need for a proper discussion. However the following points are worth noting. (1) the distances adopted by Kharchenko et al. are obtained from visual magnitudes and the reddenings from a model. They must thus be rather uncertain. (2) A certain amount of trimming is carried out (velocities further from means than $3 \sigma$ are rejected). (3) Complications in the analysis will arise when one goes to a large volume if the Galaxy is not axi-symmetrical. (4) No distinction is made in Kharchenko et al. between the SP-red and SP-blue stars discussed above and this is particularly important in the period range under discussion.

Whilst therefore the large scale picture remains unclear, it seems rather remarkable that the nearby Miras in the period range 145-200 days with radial velocities, Hipparcos proper motions and infrared photometry show a marked asymmetry in $V_{R}$. As figure 2 shows, all the stars in this group with $\left(V_{\theta} \lesssim 160 \mathrm{~km} . \mathrm{s}^{-1}\right)$ have positive values of $V_{R}$. For an axi-symmetrical galaxy there should be a symmetrical distribution of $V_{R}$ about zero in this figure at any given $V_{\theta}$. The deviation from such a distribution is sufficiently striking that it seems difficult to attribute it entirely to chance. 


\section{Acknowledgments}

I would like to thank Professor Nakada (University of Tokyo) and the organizers of this meeting for making my attendance possible. This paper depends on work done in collaboration with Patricia Whitelock.

\section{References}

Dehnen, W. (1998) AJ, 115, 2384.

Dehnen, W. (1999) ApJ, 524, L35.

Dehnen, W. (2000) AJ, 119, 800.

Feast, M.W. (1963) MNRAS, 125, 367.

Feast, M.W. and Whitelock, P.A. (1997) MNRAS, 291, 683.

Feast, M.W and Whitelock, P.A. (2000a)MNRAS, 317, 460 (paper III).

Feast, M.W. and Whitelock, P.A. (2000b) in The Evolution of the Milky Way, ed. Matteucci, F. and Giovannelli, F., Kluwer, Dordrecht, p. 229.

Fux, R. (2001) $A \& A, \mathbf{3 7 3}, 511$.

Hron, J. (1991) $A \mathscr{E} A, \mathbf{2 5 2}, 583$.

Kharchenko, N., Kilpio, E., Malkov, O. and Schilbach, E. (2002) A\&A, 384, 925.

Quillen, A.C. (2002) astro-ph 0204040.

Raboud, D., Grenon, M., Martinet, L., Fux, R. and Urdy, S. (1998) A $6 A$, 335, L61.

Whitelock, P.A. (2002) this volume.

Whitelock, P.A., Marang, F. and Feast, M.W. (2000) MNRAS, 319, 728 (paper I).

Whitelock, P.A. and Feast, M.W. (2000) MNRAS, 319, 759 (paper II).

\section{Discussion}

\section{Habing}

In your calculations of the galactic orbit, did you assume the bar is stationary? It may rotate.

Feast

For a rotating bar one needs to assume the simple elliptical orbits precess. One is then concerned with the present orientations of the Mira orbits and the bar. So the conclusions are not affected

\section{van Langevelde}

1. You find a best fit of $\phi$, the angle between the major axis of the Mira orbits and the bar, but there must be a whole range of eccentricities. What is the range of perigalactic distances?

2. What makes the 145-200 day group special? Would that indicate something about the population/age of the bar?

\section{Feast}

1. The distribution in figure 2 is essentially a distribution in eccentricity. About half the stars in that plot go within $2 \mathrm{kpc}$ of the centre. The exact perigalactic distance depends of course on the mass model.

2. That is not entirely clear. There are Miras with a range of periods in 
the bulge. At periods longer than about 200 days, the local population is dominated by variables on much more nearly circular orbits. Possibly this is because the galactic density gradient of Miras is a function of period.

Nakada

Do the bulge Miras dominate the short period (P 145-200 days) group in the solar neighbourhood?

Feast

About half the Miras in this group (SP-red stars omitted) have perigalactic distances sufficiently small that we can probably say they belong to a bulge population. However there is no evidence at present that the stars in this period group which are on more nearly circular orbits are different physically from those on highly eccentric orbits. In that sense they can perhaps all be regarded as a bulge type population. 